The Influence of Affluence), Mr. E. Paget (West Indies), and Mr. N. H. Leyland (Economic Problems). Old friends who assisted in this final conference included Prof. H. J. Fleure, who presented to the president a handsomely bound volume including typescripts of his series of annual addresses, Sir Richard Livingstone, who took the chair at the presidential address, Prof. Kenneth Mason and more than a hundred members, who paid tribute in the form of a presentation to mark the devoted work of Miss Tatton.

The main reason for the decision to wind up the Society is a tragic commentary on the post-war world. While international tourism is being en- couraged, study groups which moved so freely and were accorded unstinted help everywhere in the inter-war years are now faced with official frustration and danger of arrest as spies if they attempt to see anything not on the accepted tourist routes. In addition, increasing costs have placed such journeys beyond the resources of younger people who, if they can get grants, are required to attend meetings in their own specialisms. Few seem to have leisure for the quiet exchange of views with workers in other fields. Le Play methods in social and regional survey have, however, become basic requirements in physical and social planning all over the world.

L. Dudley Stamp

\title{
THE LACK OF THINKING IN EXAMINATIONS
}

$I_{s}^{N}$ marking examination seripts, Dr. Margaret Sutherland, lecturer in education at the Queen's University, Belfast, has noticed that many students do not deal with the topic set (The Universities Review, 32, No. 7 ; February 1960). This occurs mainly in subjects which require a basic knowledge (of facts, or of a book, theory, or other works) and some more or less intelligent discussion of some aspect of this basic knowledge. The examiner has attempted to set a question which will differ from those set in previous papers and yet allow the students to display knowledge, select relevant data and comment reasonably thereon. To this, many students pour forth a statement of basic knowledge which is mostly without relevance to the question set.

Some examiners would classify this behaviour as 'examination technique' or conscious bluffing on the part of the students who, knowing that they are unlikely to pass, have memorized answers on strictly limited topics and are going to use them no matter what the question. But not all erring students can be credited with this conscious cunning, for some irrelevant answers display consciontious and thorough preparation.

Occasionally the explanation lies in the examiner's technique; the question may have been badly worded, the interpretation which seemed obvious to the examiner was not obvious to other readers ; thus seemingly irrelevant answers may prove relevant, if the question is re-interpreted.

In other cases the weakness seems to be in the student's reading ability. The student must be technically able to read the question; but the sense of the sentence is not grasped. One word only, or part of the sentence only, dominates the rest and evokes the associations to this one element. This is an example of the so-called global thinking supposedly characteristic of early childhood, where a general concept is present or brought to mind, but finer discriminations cannot be made, and analysis, perception of detail and component parts do not occur. This leads to regression to an earlier stage of behaviour, possibly caused, like other regressions, by a stressful situation. Yet surely the concepts in question have been analysed during preparation for the examination? Surely, too, the student has been trained to attend to the different parts of meaning conveyed by whole sentences?
Does the error occur in grasping the meaning of the question or in attempting to respond to it? If it is the latter, the behaviour may be explained as regression to another primitive type of mental activity, to rote-memory in which the information sought ean be reproduced only imbedded in the impressions originally accompanying it. It is difficult to believe that, having reproduced all the data, the student still cannot select from this reproduction all that is relevant.

Yet this the student seems unable to do. It is precisely selection and discussion that are avoided. It seems increasingly probable that this avoidance may be due not to ignorance of what is wanted, not to bluff or sophisticated examination technique, not to failure in reading ability, but to emotional factors of anxiety and fear. The student approaches the examination with the feeling of not knowing enough, or of not knowing the material sufficiently well to remember it all. Acute anxiety is aroused by what seems a request to be judged on only a part of what has been learned.

Yet if the relevant material were selected and discussed, the student's answer should develop fully satisfactory dimensions. The student is invited to display a lesser amount of factual knowledge, provided evidence of thought is added.

It is possible that this avoidance by the student of thinking out a new aspect is not irrational, but the result of learning from experience. Even good examination candidates considering a new aspect of a familiar subject discover that their knowledge is inadequate; items of related knowledge do not always present themselves. The candidate has either been content to accept words without close inquiry into meaning, or has learned the accepted formulie without considering all their implications.

Even where there is no discovery of ignorance, thought does not always operate smoothly ; although we have relevant points to make, they may not all occur to us, or arrange themselves in the appropriate pattern immediately. It could be argued that students should have thought so much about the subject beforehand that their thinking has become flexible and well integrated. It remains possible that the examiner calls for discussion of an unforeseen aspect. Thus the student's resistance, conscious or not, to following a new line of thought in examination circumstances (or elsewhere) might be well founded on past experience of unsuccessful effort. It is so 
much easier to let thinking follow the usual path indicated by a signal word, or let memory take over from it.

This avoidance is to be deplored. It can be overcome at least partly by good teaching. Students who have had sufficient practice both in thinking about new aspects of their subject and in eriticizing irrelevant responses may come to recognize more clearly what is relevant and to feel some confidence in their own ability to think independently. More independent study could also help, since thinking flexibly in a subject and perceiving most of its implications are made more easy by inereasing knowledge and work in it.

\section{RESISTANCE OF GONOCOCCUS STRAINS TO PENICILLIN}

GONOCOCCUS strains isolated in 1944 were found J to be highly sensitive to penicillin. From 1946 onwards, the existence of less sensitive strains were observed. Up to the end of 1955, doses of penicillin, twice to four times as great as those which were effective against the strains isolated in 1944, were enough to stop the development of the less sensitive strains isolated at the Statens Seruminstitut, Copenhagen. Then, from a case of gonorrhoea without complications, four strains were isolated which were found to be only one-twentieth as sensitive as the 1944 strains. This discovery and the fact that the incidence of gonorrhoea remained about the same in spite of penicillin therapy indicated the need for further research. This was carried out during 1957 and 1958 by Alico Reyn and M. W. Bentzon, who made a comparison of several hundred recently isolated gonococcus strains with a hundred strains isolated in 1944 (World Health Organization Chronicle, 14 ; No. 3. March 1960). This showed that, for inhibition, about 25 per cent of the strains sent to the laboratory for diagnosis in 1957 required doses of penicillin twenty times greater than had been needed for most strains originally.

Twenty strains showing reduced sensitivity to penicillin were subcultured for $30-90$ days on a medium with no penicillin, without any increase in sensitivity being produced.

Both in 1957 and 1958, some 200 strains were tested for penicillin sensitivity by the tablet method, that is to say, by placing penicillin tablets in the culture medium and measuring the zone of inhibition around them. The zone diameters varied between 20 and
$50 \mathrm{~mm}$. In both years, about 39 per cent of the strains showed reduced sensitivity, that is to say, zone diameters of $34 \mathrm{~mm}$. or less. In 1958, however, the number of strains with zone diameters of $44 \mathrm{~mm}$. or more was significantly lower than in 1957, which seems to indicate that the selection (and induction) of the strains with a comparatively reduced penicillin sensitivity continues.

None of the 1957 strains was completely resistant to streptomycin, but two of the 1958 strains (from the same patient) were.

In both the 1957 and 1958 strains a significant positive correlation was observed between sensitivity to penicillin and sensitivity to streptomycin.

From June 1957 onwards, atypical gonococcus strains-most of them sensitive to penicillinappeared in the cultures. The growth of these strains on the routine media is poor; they require special conditions for culture and are able to ferment glucose only weakly or not at all. Serological examination showed them to be distinguished from normal strains by the frequent presence of anti-complementary antigens. The proportion of atypical strains rose to 40 per cent in January 1958 when the culture and fermentation media were changed. The change of culture medium had yet another effect: the inhibition zones of the atypical strains-and of these alone-were greater on ox-heart broth than on oxmeat broth. This phenomenon has not yet been explained. It is possible that the emergence of atypical strains is related to the mode of action of the penicillin.

\section{GLOBULAR CLUSTERS}

A STRONOMY has always suffered because of the uneven distribution of its personnel and equipment between the northern and southern hemispheres. Especially welcome, therefore, are the growing number of contributions from the Radeliffe Observatory at Pretoria. A series of three contributions by Dr. T. D. Kinman (Mon. Not. Roy. Astro. Soc., 119, $157,538,559 ; 1959)$ represents an important addition to our knowledge of globular clusters. A study of their motions in the Galaxy is of interest because these clusters are prototypes of Baade's Population II and are believed to contain the oldest stars in the Galaxy. Kinman has obtained the radial velocities of thirty southern globular clusters, for sixteen of which no previous determinations of velocities existed; velocities are now available for seventy clusters in all, more than two-thirds of the known clusters in our Galaxy. The effect of the metal/hydrogen ratio on the strengths of the spectral lines of metallic elements in the clusters was studied by Kinman. He found that this ratio varies from
0.1 to less than 0.01 for different clusters. He also found that clusters of the earliest spectral type, the stars of which have a very low abundance of metal, form an extended spherical distribution with little concentration in the galactic plane, while those of latest type, the stars of which have the highest abundance of metal, show a strong concentration in the galactic plane. Clusters of intermediate tvpe show an intermediate distribution.

The motion of the Sun relative to the globular clusters was determined; the apex of the solar motion does not deviate from the direction of the Sun's galactic rotation. The new analysis confirms the older conclusion that the orbits of the individual clusters in the Galaxy are highly eccentric. The mean angular momentum per unit mass of the cluster system is the same as the mean for the rest of the Galaxy. This is in accord with the hypothesis that the clusters were formed in a spherical gas cloud which was in the process of contracting to form the galactic disk. 